\title{
Responses of Parasitoids to Volatiles Induced by Chilo partellus Oviposition on Teosinte, a Wild Ancestor of Maize
}

\author{
Daniel M. Mutyambai ${ }^{1,3,4} \cdot$ Toby J. A. Bruce ${ }^{2} \cdot$ Charles A. O. Midega $^{1} \cdot$ \\ Christine M. Woodcock ${ }^{2} \cdot$ John C. Caulfield ${ }^{2}$. Johnnie Van Den Berg ${ }^{3} \cdot$ \\ John A. Pickett ${ }^{2}$ - Zeyaur R. Khan ${ }^{1,3}$
}

Received: 18 November 2014 / Revised: 19 February 2015 / Accepted: 23 February 2015 / Published online: 6 May 2015

(C) The Author(s) 2015. This article is published with open access at Springerlink.com

\begin{abstract}
Maize, a genetically diverse crop, is the domesticated descendent of its wild ancestor, teosinte. Recently, we have shown that certain maize landraces possess a valuable indirect defense trait not present in commercial hybrids. Plants of these landraces release herbivore-induced plant volatiles (HIPVs) that attract both egg [Trichogramma bournieri Pintureau \& Babault (Hymenoptera: Trichogrammatidae)] and larval [Cotesia sesamiae Cameron (Hymenoptera: Braconidae)] parasitoids in response to stemborer egg deposition. In this study, we tested whether this trait also exists in the germplasm of wild Zea species. Headspace samples were collected from plants exposed to egg deposition by Chilo partellus Swinhoe (Lepidoptera: Crambidae) moths and unexposed control plants. Four-arm olfactometer bioassays with parasitic wasps, T. bournieri and C. sesamiae, indicated that both egg and larval parasitoids preferred HIPVs from plants with eggs in four of the five teosinte species sampled. Headspace samples from oviposited plants released higher amounts of EAG-active compounds such as (E)-4,8-dimethyl-1,3,7-nonatriene. In oviposition choice bioassays, plants without eggs were significantly preferred for subsequent oviposition by moths compared to plants with prior oviposition.
\end{abstract}

Toby J. A. Bruce

toby.bruce@rothamsted.ac.uk

1 Habitat Management Programme, International Centre of Insect Physiology and Ecology, P.O Box 30-40305, Mbita, Kenya

2 Department of Biological Chemistry, Rothamsted Research, Harpenden, Herts AL5 2JQ, UK

3 Unit for Environmental Sciences and Management, North-West University, Potchefstroom 2520, South Africa

4 Zoological Services, Veterinary Department, Ministry of Agriculture, Livestock and Fisheries, P.O. Box 30028-00100, Nairobi, Kenya
These results suggest that this induced indirect defence trait is not limited to landraces but occurs in wild Zea species and appears to be an ancestral trait. Hence, these species possess a valuable trait that could be introgressed into domesticated maize lines to provide indirect defense mechanisms against stemborers.

Keywords Induced defense - oviposition · plant volatiles . tritrophic interactions $\cdot$ crop wild relative

\section{Introduction}

Emission of herbivore-induced volatiles (HIPVs) in response to herbivore feeding damage has been studied intensively during the past two decades. Recently, a relatively small but growing number of investigations have revealed that oviposition can induce production of plant volatiles that attract parasitoids (Bruce et al. 2010; Colazza et al. 2004; Fatouros et al. 2012; Hilker and Meiners 2006; Tamiru et al. 2011, 2012). Detection of egg deposition and subsequent changes in chemical phenotype prepares plants for the impending attack by emerging phytophagous larvae (Bruce et al. 2010; Hilker et al. 2002). Natural enemies of herbivores make use of these HIPVs for host location (Dicke and Sabelis 1988; Powell, et al. 1998; Turlings et al. 1990), but different plant species release entirely different HIPV blends, and, even within one plant species, there are clear differences among genotypes (Gouinguené et al. 2001; Takabayashi et al. 1991; Turlings et al. 1998).

Information regarding variability in HIPV emission comes mainly from studies of cultivated plants (Gouinguené et al. 2001; Krips 2000; Takabayashi et al. 1991; Turlings et al. 1998). Studies of wild systems include a wild cotton variety that was found to release much higher quantities of induced volatiles than cultivated varieties (Loughrin et al. 1995), and 
of larval regurgitant applied to mechanically damaged maize leaves (Gouinguené et al. 2001). However, no studies of tritrophic interactions have yet investigated wild maize following egg deposition by a herbivore. For a better understanding of the ecological relevance and evolutionary history of oviposition-induced plant signalling, it is necessary to study these signals in wild systems.

The spotted stemborer, Chilo partellus (Swinhoe) (Lepidoptera: Crambidae) is one of the most damaging lepidopteran pests of maize in eastern and southern Africa and Asia, causing yield losses of up to $88 \%$ (Kfir et al. 2002). Effective chemical control is difficult, mostly due to the protection provided to larvae by plants when feeding inside plant whorls and stems (Slabbert and Van den Berg 2009). Furthermore, insecticides are not economical for smallholder farmers. Thus, the ecology of tritrophic interactions presents an opportunity for development of crop protection approaches that make use of induced innate plant defenses.

For better understanding and utilization of indirect defense traits in pest management strategies, we need to consider their evolutionary and ecological history when subjected to forces such as domestication or breeding. A recent review states that further research into pest resistance of maize wild ancestors, the teosintes, is needed (de Lange et al. 2014). The objectives of this study were, therefore, to determine: (1) volatile profile changes in response to $C$. partellus egg deposition on wild maize; (2) behavioral responses of parasitoids to HIPVs collected from wild maize exposed to stemborer oviposition; and (3) the effect of oviposition-induced volatiles on subsequent moth oviposition on these plants.

\section{Methods and Materials}

Plants Seeds of the following teosinte species were obtained from the International Maize and Wheat Improvement Centre (CIMMYT), Mexico: Zea diploperennis Iltis, Doebley \& Guzman, Z. huehuetenangensis (Iltis \& Doebley) Doebley, Z. mays spp. mexicana (Schrader) Iltis, Z. m. spp. parviglumis Iltis \& Doebley, and Z. perennis (Hitcht.) Reeves \& Manglesdorf. Seeds were grown individually in pots filled with fertilized soil in an insect-proof screen house at the icipeThomas Odhiambo campus, Mbita point $\left(0^{\circ} 25^{\prime} \mathrm{S}, 34^{\circ} 12^{\prime} \mathrm{E}\right.$; $1200 \mathrm{~m}$ above sea level), in Western Kenya. All seedlings were grown under natural conditions $\left(25^{\circ} \mathrm{C}, 65 \% \mathrm{RH}\right.$; $12 \mathrm{~L}$ : 12D) and used in the experiments when 3-4 wk-old.

Insects Chilo partellus moths were obtained from the insect mass rearing unit at the icipe-Thomas Odhiambo campus. The larvae originated from field-collected stemborers, principally from sorghum (Sorghum bicolor L. Moench) fields in the Mbita region. Larvae were reared on a semi-synthetic diet containing sorghum leaf powder (Ochieng et al. 1985). Field collected egg parasitoids, Trichogramma bournieri Pintureau \& Babault (Hymenoptera: Trichogrammatidae) and larval parasitoids, Cotesia sesamiae Cameron (Hymenoptera: Braconidae), were reared on stemborer eggs and larvae, respectively, using methodologies described by Overholt et al. (1994). The experimental insects were maintained at $24 \pm 3{ }^{\circ} \mathrm{C}$, $70 \pm 5 \%$ RH, 12L: $12 \mathrm{D}$.

Chemicals Authentic chemical standards ( $>95 \%$ purity) of (E)-2-hexenal, (Z)-3-hexen-1-ol, (Z)-3-hexenyl acetate, $(E, E)$ 2,4-heptadienal, (Z)-2-heptenal, hexanal, decanal, nonane, $\alpha$ pinene, 2,3-butanediol, myrcene, limonene, 6-methyl-5hepten-2-one, 3,4-dimethylacetophenone, and $(E, E)$-farnesyl chloride were purchased from Sigma Aldrich (Gillingham, UK). (E)- $\beta$-Farnesene ( $99 \%$ pure by GC) was synthesized from $(E, E)$-farnesyl chloride (Kang et al. 1987). (E)-4,8Dimethyl-1,3,7-nonatriene ( $>98 \%$ ) was synthesised from geraniol by oxidation to the aldehyde followed by Wittig methylenation (Leopold 1990).

Volatile Organic Compound (VOC) Collection Headspace sampling (Agelopoulos et al. 1999) was used to collect volatile compounds from whole maize plants, with and without stemborer eggs. Prior to volatile collection, seedlings were placed in oviposition cages $(80 \times 40 \times 40 \mathrm{~cm})$ into which five gravid naïve female stemborer moths were introduced and kept overnight for oviposition. A wad of cotton wool $(10 \mathrm{~cm}$ diam) moistened with water was placed into the cage for moths to feed on. Control plants were kept inside similar cages but without $C$. partellus moths. Volatiles were collected from these plants for a period of $48 \mathrm{~h}$, starting at the last $2 \mathrm{~h}$ of the photophase of the following day. Leaves with or without eggs were enclosed in polyethyleneterephthalate (PET) bags (3.2 L, $12.5 \mathrm{~mm}$ thickness) heated to $150{ }^{\circ} \mathrm{C}$ before use, and fitted with a swagelock inlet and outlet ports. Charcoal-filtered air was pumped $\left(600 \mathrm{ml} \mathrm{min}^{-1}\right)$ through the inlet port. Volatiles were collected on Porapak Q (0.05 g, 60/80 mesh; Supelco) filters inserted into the outlet through which air was drawn at $400 \mathrm{ml}$ $\mathrm{min}^{-1}$. Elution of the entrained volatiles was done using $0.5 \mathrm{ml}$ dichloromethane. The eluted samples were stored in tightly capped microvials in a $-20{ }^{\circ} \mathrm{C}$ freezer prior to bioassays and further analysis. Entrainments from both oviposited and control plants were replicated four times, and each plant was used only once.

Four-arm Olfactometer Bioassay Responses of parasitoids to plant derived volatiles were tested in a Perspex four-arm olfactometer (Pettersson 1970). A choice-test was carried out to compare insect responses to headspace samples from oviposited and control plants. Two opposing arms held the test stimuli ( $10 \mu$ aliquots of headspace sample). The remaining two arms held solvent controls. Olfactometers were used only once for each replicate of the bioassay, and they were 
washed and air dried before the next test. The experiment was replicated 12 times. Headspace samples $(10 \mu \mathrm{l}$ aliquots) were applied, using a micropipette (Drummond 'microcap', Drummond Scientific Co., Broomall, PA, USA), to a piece of filter paper $(4 \times 25 \mathrm{~mm})$ placed in the inlet port at the end of each olfactometer arm. To avoid bias, the position of the treatments was randomly re-allocated between each tests, and the olfactometer was rotated after every $4 \mathrm{~min}$ during the test. One-day-old gravid female parasitoids without any prior exposure to plants or hosts were isolated from the rearing cage and allowed to acclimatize to room temperature for $1 \mathrm{~h}$. They were transferred individually into the central chamber of the olfactometer using a custom-made piece of glass tubing. Air was drawn through the four arms towards the centre at $260 \mathrm{ml}$ $\mathrm{min}^{-1}$. The time spent by parasitoids in each olfactometer arm was recorded with 'Olfa' software (F. Nazzi, Udine, Italy) for $12 \mathrm{~min}$.

Gas Chromatography (GC) Analysis Entrained VOCs were analyzed using a Hewlett-Packard 7890 GC machine (Agilent Technologies) equipped with a cool-on column injector, a non-polar HP-1 capillary column $(50 \mathrm{~m}, 0.32 \mathrm{~mm}$ internal diam, $0.52 \mu \mathrm{m}$ film thickness) and a flame ionization detector (FID). Four $\mu \mathrm{l}$ of headspace sample were injected into the injector port of the GC instrument. The oven temperature was maintained at $30^{\circ} \mathrm{C}$ for $2 \mathrm{~min}$ and then programmed at $5{ }^{\circ} \mathrm{C}$ min- 1 to $250{ }^{\circ} \mathrm{C}$. The carrier gas was hydrogen. Data were analyzed using HP Chemstation software. Quantification was done by dividing peak areas by known amounts of external standards. The emission rate in terms of ng plant ${ }^{-1} \mathrm{~h}^{-1}$ was obtained by multiplying inverse of the proportion of the total headspace used and dividing by the number of hours in the sampling period. Four replicate headspace samples were analyzed for each treatment.

Coupled GC-Electroantennography (GC-EAG) Analysis GC-EAG was carried out using the antennae of female $C$. sesamiae with the headspace samples of the teosinte species that had elicited positive responses during olfactometer bioassays. The GC-EAG system, in which the effluent from the GC column is simultaneously directed to the antennal preparation and the GC detector, was described previously by Wadhams (1990). EAG recordings were made using Ag-AgCl glass electrodes filled with saline solution, as described by Maddrell (1969) but without glucose. A female parasitoid was chilled for $1 \mathrm{~min}$, and the head was excised, and the tips of both antennae were removed to ensure a good contact in the recording electrode. The indifferent electrode was placed within the head capsule. Signals were passed through a high impedance amplifier (UN-06; Syntech, Hilversum, The Netherlands) and analyzed using a customized Syntech software package. Separation of volatiles was done on a 6890 N GC (Agilent Technologies) equipped with a cold on- column injector and a FID using an HP-1 column (50 m, $0.32 \mathrm{~mm}$ internal diam, $0.52 \mu \mathrm{m}$ film thickness). The oven temperature was maintained at $30^{\circ} \mathrm{C}$ for $2 \mathrm{~min}$ and then programmed at $15{ }^{\circ} \mathrm{C} \min ^{-1}$ to $250{ }^{\circ} \mathrm{C}$. The carrier gas was helium. Outputs from the EAG amplifier and the FID were analyzed using Syntech software.

Coupled GC-Mass Spectrometry (GC-MS) Analysis Aliquots of attractive headspace samples were analyzed using an Hewlett-Packard 5890 GC machine (Agilent Technologies) equipped with an HP-1 column $(50 \mathrm{~m}$, $0.32 \mathrm{~mm}$ internal diam, $0.52 \mu \mathrm{m}$ film thickness) directly coupled to a mass spectrometer (VG Autospec; Fisons Instruments, Manchester, UK) equipped with a cool oncolumn injector. Ionization was performed by electron impact $\left(70 \mathrm{eV}\right.$ at $\left.250^{\circ} \mathrm{C}\right)$. The oven temperature was maintained at $30{ }^{\circ} \mathrm{C}$ for $5 \mathrm{~min}$ and then programmed at $5{ }^{\circ} \mathrm{C} \mathrm{min}^{-1}$ to $250{ }^{\circ} \mathrm{C}$. Tentative identifications were made by comparison of mass spectra with those collected in mass spectral databases (NIST 2005). Tentative identifications of the compounds were confirmed through co-injections with authentic standards.

Oviposition Bioassay Two-choice tests were conducted using modifications of the methodology of Khan et al. (2007) in oviposition cages $(80 \times 40 \times 40 \mathrm{~cm})$ covered by fine cloth mesh netting with a cloth access flap. Prior to the twochoice test, a 3-4-wk-old potted maize plant was caged overnight with five gravid naïve $C$. partellus moths to allow oviposition. A wad of cotton wool (10 $\mathrm{cm}$ diam) moistened with water was introduced into the cage for the moths to feed on. After $24 \mathrm{~h}$, the positions of egg batches on leaves were marked. The following day, another maize plant of the same species and age but without prior exposure to moths was placed into each of the oviposition cages, adjacent to the previously exposed plant. Thus, each cage had two potted maize plants positioned at opposite sides, one exposed to moths the day before, and the other without any prior exposure to moths. Five new gravid naive $C$. partellus moths then were introduced into the cage and allowed to oviposit for $48 \mathrm{~h}$ under natural conditions of approximately L12:D12. Plants were removed, and the number of newly laid eggs and egg batches on each plant were counted under a light binocular microscope. 'Preference' was taken in this context to be differential oviposition on a plant when the insect is given a choice between two plants of the same variety but with different treatments. Data collected were expressed as the mean proportion (percentage) of total number of eggs oviposited during the second oviposition period on plants in the two-choice test. This experiment was replicated 10 times.

Statistical Analyses Statistical analyses were done using R software (version 3.0.2). Time spent in each arm of the fourarm olfactometer bioassay was compared by analysis of 
variance (ANOVA) after conversion of the data into proportions of the total time the insect was allowed to make its choice (12 $\mathrm{min}$ ), followed by a log ratio transformation to allow analysis of compositional data (Aitchison 1981; Tamiru et al. 2011). Means were separated using the Tukey test with $\alpha$ set at 0.05 . The two-sample (unpaired) student's $t$-test was used to determine if there were any differences between the numbers of eggs and egg batches laid on plants that were previously either exposed or non-exposed to oviposition.

\section{Results}

Behavioral Responses of Parasitoids to Headspace Samples of VOCs Female T. bournieri individuals spent significantly more time in olfactometer arms containing volatiles from plants exposed to oviposition in comparison to those with volatiles from plants without eggs and solvent controls, for four of the five teosinte species ( $Z$. huehuetenangensis $F_{2,33}=6.505, P=0.004 ; Z$. $m$. spp. mexicana $F_{2,33}=4.41, P=$
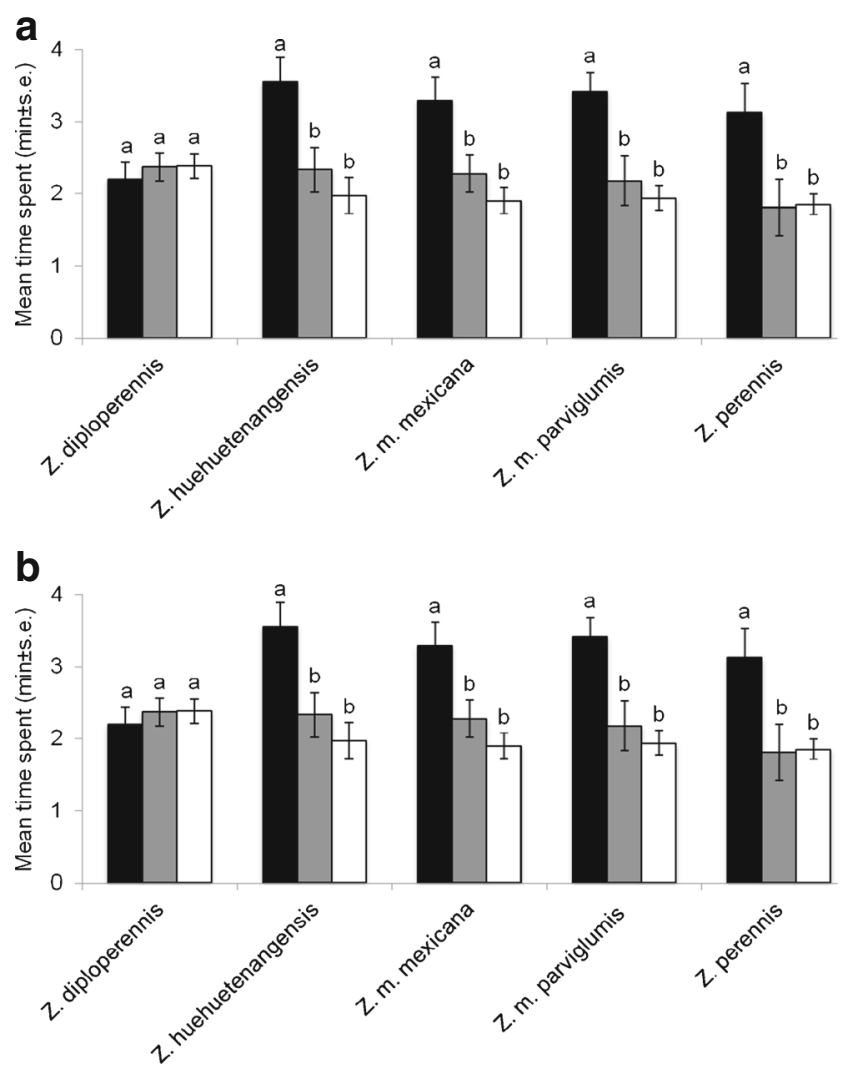

Fig. 1 Behavioral response of female parasitoids to volatiles collected from teosinte with C. partellus eggs (exposed), without Chilo partellus eggs (non-exposed) and solvent control in a four-arm olfactometer bioassay. (a) response of Trichogramma bournieri; (b) response of Cotesia sesamiae. Each female parasitoid was observed for $12 \mathrm{~min}(N=$ 12). Bars followed by different letters are significantly different at $P<0.01$

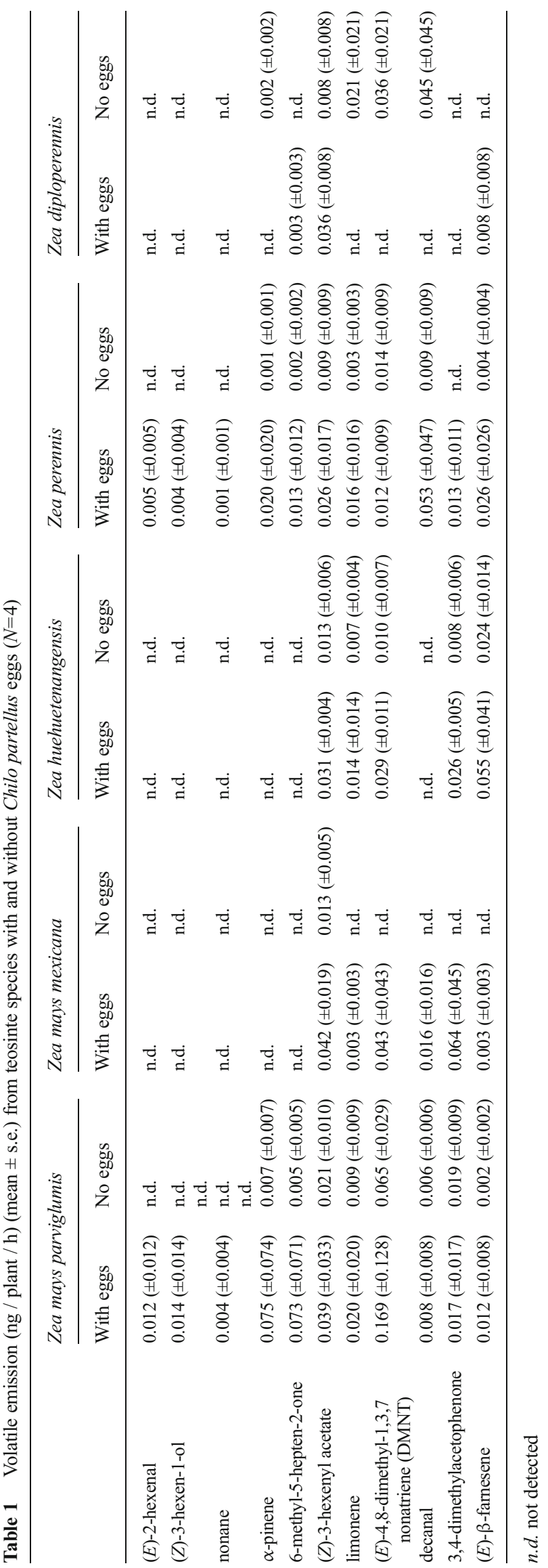




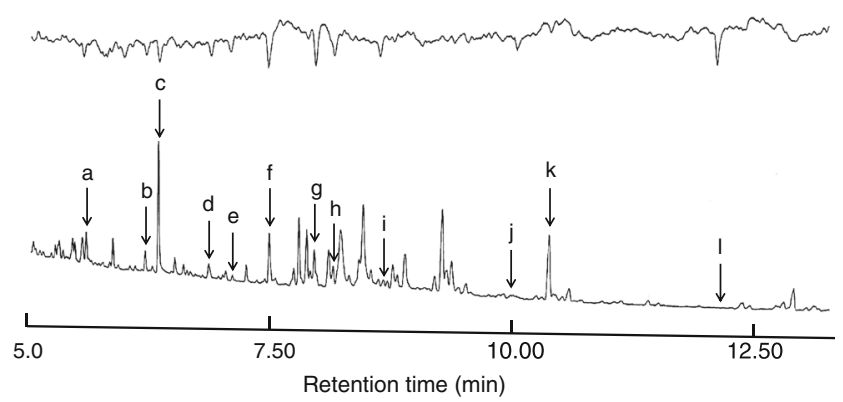

Fig. 2 A representative GC-EAG response of female Cotesia sesamiae to volatiles collected from Zea perennis with eggs. FID peaks marked are those which elicited antennal response in coupled runs: $a=$ hexanal, $b=$ 2,3-butanediol, $\mathrm{c}=(E)$-2-hexenal, $\mathrm{d}=(Z)$-3-hexen-1-ol, $\mathrm{e}=$ nonane, $\mathrm{f}=$ (Z)-2-heptenal, $\mathrm{g}=6$-methyl-5-heptene-2-one, $\mathrm{h}=(E, E)$-2,4-heptadienal, $\mathrm{i}=$ limonene, $\mathrm{j}=(E)$-4,8-dimethyl-1,3,7-nonatriene (DMNT), $\mathrm{k}=$ decanal, $1=(E)-\beta$-farnesene

$0.020 ; Z$. $m$. spp. parviglumis $F_{2,33}=7.357, P=0.002$; and $Z$. perennis $F_{2,33}=6.492, P=0.004$ ) (Fig. 1a). Similar results were observed for larval parasitoids, Cotesia sesamiae (Z. huehuetenangensis $F_{2,33}=8.428, P=0.001 ; Z$. m. spp. mexicana $F_{2,33}=10.15, P<0.001 ; Z$. $m$. spp. parviglumis $F_{2,33}=10.15, P<0.001 ; Z$. perennis $F_{2,33}=5.488, P=0.009$ ) (Fig. 1b). In the case of $Z$. diploperennis, there were no significant differences in time spent in arms with volatiles from plants exposed to egg deposition, plants without eggs and solvent controls for both egg and larval parasitoids $\left(F_{2,33}=\right.$ $0.434, P=0.651 ; F_{2,33}=0.391, P=0.679$, respectively).

\section{Comparison of Volatiles Emitted from Plants with and} Without Eggs GC analysis revealed quantitative and qualitative changes in the volatile blend profile emitted by plants exposed to egg deposition (Table 1). Zea mays parviglumis, Zea mays mexicana, and Zea perennis emitted more EAG active compounds when exposed to $C$. partellus oviposition compared to unexposed plants.
Identification of Attractive Volatile Organic Compounds GC-EAG recordings with the attractive samples from teosinte species and subsequent GC-MS identification showed that $C$. sesamiae antennae were responsive to hexanal, 2,3-butanediol, (E)-2-hexenal, (Z)-3-hexen-1-ol, nonane, (Z)2-heptenal, 6-methyl-5-heptene-2-one, $(E, E)$-2,4-heptadienal, limonene, $(E)$-4,8-dimethyl-1,3,7-nonatriene (DMNT), decanal, and (E)- $\beta$-farnesene (Fig. 2).

Oviposition Preference In two-choice tests, a significantly higher number of eggs was laid on unexposed control plants in comparison to plants with prior egg deposition, except for Z. diploperennis (Table 2). Similarly, a higher number of egg batches and number of eggs per batch were laid in $Z$. huehuetenangensis, $Z$. mays spp. mexicana, Z. $m$. spp. parviglumis, and $Z$. perennis, although the difference was only significant in $Z$. $m$. spp. parviglumis $(P=0.04)$ (Table 2).

\section{Discussion}

Our findings provide evidence that indirect defense involving insect egg-induced HIPV emission is an ancestral trait in maize, present in several species of teosinte. Our previous study (Tamiru et al. 2011) showed that the egg-induced HIPV emission trait was present in certain landraces, but not in the commercial hybrid maize varieties tested to date.

Within the teosinte species, there was variation in the quality and quantity of volatiles emitted following egg deposition. Noticeably, Z. m. spp. parviglumis, which is considered the closest relative to cultivated maize (Doebley and Wang 1997; Kellogg 1997), produced more HIPVs after egg deposition, whereas $Z$. diploperennis showed little change in volatile emission. (E)-4,8-Dimethyl-1,3,7-nonatriene (DMNT), a key

Table 2 Percentages of Chilo partellus eggs laid per plant ( \pm SEM), number of egg batches per plant $( \pm$ SEM), and number of eggs per egg batch $( \pm$ SEM) for five teosinte species exposed and unexposed to prior egg deposition

\begin{tabular}{|c|c|c|c|}
\hline $\begin{array}{l}\text { Teosinte species: oviposited (T) } \\
\text { vs. unoviposited }(\mathrm{C})\end{array}$ & $\begin{array}{l}\text { Mean } \% \text { of } \\
\text { eggs per plant }\end{array}$ & $\begin{array}{l}\text { Mean number of egg } \\
\text { batches per plant }\end{array}$ & $\begin{array}{l}\text { Mean number } \\
\text { of eggs per batch }\end{array}$ \\
\hline Zea diploperennis $(\mathrm{T})$ & $50.79( \pm 9.17) \mathrm{a}$ & $2.83( \pm 0.65) \mathrm{a}$ & $25.92( \pm 4.42) \mathrm{a}$ \\
\hline Z. diploperennis (C) & $49.21( \pm 9.17) \mathrm{a}$ & $3.00( \pm 0.52) \mathrm{a}$ & $25.10( \pm 8.93) \mathrm{a}$ \\
\hline Z. huehuetenangensis $(\mathrm{T})$ & $45.99( \pm 2.65) \mathrm{a}$ & $9.89( \pm 0.98) \mathrm{a}$ & $34.62( \pm 3.08) \mathrm{a}$ \\
\hline Z. huehuetenangensis (C) & $54.01( \pm 2.65) \mathrm{b}$ & $10.33( \pm 0.73) \mathrm{a}$ & $36.62( \pm 5.58) \mathrm{a}$ \\
\hline Z. mays spp. mexicana $(\mathrm{T})$ & $37.43( \pm 6.10) \mathrm{a}$ & $3.90( \pm 0.98) \mathrm{a}$ & $30.72( \pm 4.76) \mathrm{a}$ \\
\hline Z. mays spp. mexicana $(\mathrm{C})$ & $62.57( \pm 6.10) b$ & $6.40( \pm 0.86) \mathrm{a}$ & $38.92( \pm 8.90) \mathrm{a}$ \\
\hline Z. m. spp. parviglumis $(\mathrm{T})$ & $36.46( \pm 6.83) \mathrm{a}$ & $4.50( \pm 1.07) \mathrm{a}$ & $29.60( \pm 2.96) \mathrm{a}$ \\
\hline Z. m. spp. parviglumis $(\mathrm{C})$ & $63.54( \pm 6.83) b$ & $7.63( \pm 1.31) \mathrm{a}$ & $37.02( \pm 6.32) b$ \\
\hline Z. perennis $(\mathrm{T})$ & $34.10( \pm 6.79) \mathrm{a}$ & $10.17( \pm 2.40) \mathrm{a}$ & $20.30( \pm 2.76) \mathrm{a}$ \\
\hline Z. perennis $(\mathrm{C})$ & $65.90( \pm 6.70) \mathrm{b}$ & $13.00( \pm 2.73) \mathrm{a}$ & $32.77( \pm 5.90) \mathrm{a}$ \\
\hline
\end{tabular}

Values in a column (between each oviposited and unoviposited teosinte species), followed by the same letter, are not significantly different at $P=0.05$ (two-sample $t$-test) 
semiochemical known to attract $C$. sesamiae larval parasitoids (Khan et al. 1997), was released in larger amounts in three of the teosinte species exposed to egg deposition: $Z$. $m$. spp. mexicana, Z. m. spp. parviglumis, and Z. perennis. There was some overlap in the blends of EAG active egg induced volatiles emitted by teosinte species and the maize landraces we studied previously (Tamiru et al. 2011, 2012), with compounds such as DMNT, decanal, and $(E)-\beta$-farnesene being emitted by both. However, there were also differences, for example, methyl salicylate and TMTT were more important in the landraces, and 6-methyl-5-heptene-2-one occurred in the teosinte species. Interspecific as well as intraspecific variation in HIPV emission following larval damage of different maize breeding lines, as well as teosinte varieties, has been reported (Degen et al. 2004; Gouinguené et al. 2001), However, egg induced effects have not previously been investigated in wild maize species.

In behavioral bioassays, in four of the five species tested, both egg and larval parasitoids preferred volatiles from plants exposed to egg deposition compared to those from unexposed plants. The only species for which this effect was not observed was $Z$. diploperennis, the teosinte species that showed no induction of volatiles after exposure to oviposition and possibly a decrease. The attraction of both egg and larval parasitoids is considered a preventive defense strategy since larval parasitoids are recruited in advance, before the phytophagous larvae emerge from eggs and start causing plant damage (Bruce et al. 2010). Conversely, the herbivore itself avoided egg exposed plants. Gravid C. partellus moths preferred to oviposit on teosinte plants with no prior exposure to egg deposition in the four of the five species for which a change in HIPV profile was observed. However, there was no preferential oviposition behavior observed for $Z$. diploperennis. We hypothesize that the presence of volatiles attractive to the herbivore's natural enemies could have influenced oviposition behavior, or that moths could have used this as a mechanism to avoid intraspecific competition. Previous studies have shown that HIPVs may indicate pressure from natural enemies and the risk of competition for resources on plants emitting HIPVs, resulting in female moths avoiding these plants (Dicke and Baldwin 2010; Heil and Karban 2010; Zakir et al. 2013).

The current results showed that oviposition-induced plant signalling is an ancestral trait in maize that exists even in predomestication germplasm. This valuable indirect defense trait is, therefore, not limited to the Zea mays landraces in which we originally discovered the trait (Tamiru et al. 2011). The observation of egg-induced HIPV emission in wild Zea species and landraces, but not in the commercial hybrids investigated to date, suggests that breeding for yield and palatability could have resulted in the loss of secondary defense metabolites in improved maize breeding lines (Benrey et al. 1998). The latter were developed primarily with the aim of higher yield and improved grain quality. In hybrid breeding programmes, pesticide applications often are used to protect breeding lines, which may have resulted in elite maize breeding material losing some of its natural defense traits. However, smallholder farmers in Africa often do not have access to insecticides, which means that when crops are grown under unprotected conditions, the loss of natural plant resistance traits, such as the indirect defense trait described here, can lead to yield instability due to crop losses caused by insect pests.

Acknowledgments We are grateful to CIMMYT-Mexico office for providing different wild maize varieties. We thank Amos Gadi, Isaac Odera, Silas Ouko, Kennedy Omendo, and Daniel Simiyu for insect rearing, technical assistance, and screen house operations, and Daisy Salifu for statistical advice. Financial support was from German Academic Exchange (DAAD) fellowship to Daniel Mutyambai and European Union funded ADOPT project DCI-FOOD.2010/230224. This work was supported by the BBSRC-SCPRID (BB/J011371/1) project. Rothamsted Research receives grant-aided support from Biotechnology and Biological Sciences Research Council (BBSRC).

Open Access This article is distributed under the terms of the Creative Commons Attribution 4.0 International License (http:// creativecommons.org/licenses/by/4.0/), which permits unrestricted use, distribution, and reproduction in any medium, provided you give appropriate credit to the original author(s) and the source, provide a link to the Creative Commons license, and indicate if changes were made.

\section{References}

Agelopoulos NG, Hooper AM, Maniar SP, Pickett JA, Wadhams LJ (1999) A novel approach for isolation of volatile chemicals released by individual leaves of a plant in situ. J Chem Ecol 25:1411-1425

Aitchison J (1981) A new approach to null correlations of proportions. J Int Assoc Math Geol 13:175-189

Benrey B, Callejas A, Rios L, Oyama K, Denno RF (1998) The effects of domestication of Brassica and Phaseolus on interactions between phytophagous insects and parasitoids. Biol Control 11:130-140

Bruce TJA, Midega CAO, Birkett MA, Pickett JA, Khan ZR (2010) Is quality more important than quantity? Insect behavioural responses to changes in a volatile blend after stemborer oviposition on an African grass. Biol Lett 6:314-317

Colazza S, Furarino A, Peri E, Salerno G, Conti E, Bin F (2004) Insect oviposition induces volatile emission in herbaceous plants that attract egg parasitoids. J Exp Biol 207:47-53

de Lange ES, Balmer D, Mauch-Mani B, Turlings TCJ (2014) Insect and pathogen attack and resistance in maize and its wild ancestors, the teosintes. New Phytol 204:329-341

Degen T, Dillmann C, Marion-Poll F, Turlings TCJ (2004) High genetic variability of herbivore-induced volatile emission within a broad range of maize inbred lines. Plant Physiol 135:1928-1938

Dicke M, Baldwin IT (2010) The evolutionary context for herbivoreinduced plant volatiles: beyond the "cry for help". Trends Plant Sci 15:167-175

Dicke M, Sabelis MW (1988) How plants obtain predatory mites as bodyguards. Neth J Zool 38:148-165

Doebley J, Wang RL (1997) Genetics and the evolution of plant form: an example from maize. Cold Spring Harb Symp Quant Biol 62:361367

Fatouros NE, Lucas-Barbosa D, Weldegeris BT, Pashalidou FG, van Loon JJA, Dicke M, Harvey JA, Gols R, Huigens ME (2012) 
Plant volatiles induced by herbivore egg deposition affect insects of different trophic levels. PLoS One 7: e43607

Gouinguené S, Degen T, Turlings TCJ (2001) Variability in herbivoreinduced odour emissions among maize cultivars and their wild ancestors (teosinte). Chemoecology 11:9-16

Heil M, Karban R (2010) Explaining evolution of plant communication by airborne signals. Trends Ecol Evol 25:137-144

Hilker M, Meiners T (2006) Early herbivore alert: insect eggs induce plant defense. J Chem Ecol 32:1379-1397

Hilker M, Kobs C, Varama M, Schrank K (2002) Insect egg deposition induces Pinus sylvestris to attract egg parasitoids. J Exp Biol 205: 455-461

Kang SK, Chung GY, Lee DH (1987) A convenient synthesis of (E)- $\beta$ farnesene. Bull Kor Chem Soc 8:351-353

Kellogg EA (1997) Plant evolution: the dominance of maize. Curr Biol 7: 411-413

Kfir R, Overholt WW, Khan ZR, Polaszek A (2002) Biology and management of economically important lepidopteran cereal stem borers in Africa. Annu Rev Entomol 47:701-731

Khan ZR, Ampong-Nyarko K, Chilishwa P, Hassanali A, Kimani S, Lwande W, Overholt WA, Pickett JA, Smart LE, Woodcock CM (1997) Intercropping increases parasitism of pests. Nature 388: 631-632

Khan ZR, Midega CAO, Wadhams LJ, Pickett JA, Mumuni A (2007) Evaluation of Napier grass (Pennisetum purpureum) varieties for use as trap plants for the management of African stemborer (Busseola fusca) in a push-pull strategy. Entomol Exp Appl 124: 201-211

Krips OE (2000) Plants effects on biological control of spider mites in the ornamental crop gerbera. PhD Thesis, Wageningen University, Netherlands. Pp. 79-92

Leopold EJ (1990) Elective hydroboration of a 1,3,7-triene: homogeraniol. Org Synth 64:164-171

Loughrin JH, Manukian A, Heath RR, Tumlinson JH (1995) Volatiles emitted by different cotton varieties damaged by feeding beet armyworm larvae. J Chem Ecol 21:1217-1227

Maddrell, SHP (1969) Secretion by the Malphigian tubules of Rhodnius. The movement of ions and water. J Exp Biol 51: 71-97

Nist (2005) NIST mass spectral search for the NIST/EPA/NIH mass spectral library version 2.0. office of the Standard Reference Data Base, National Institute of Standards and Technology, Gaithersburg, Maryland
Ochieng RS, Onyango FO, Bungu MDO (1985) Improvement of techniques for mass-culture of Chilo partellus (Swinhoe). Insect Sci Appl 6:425-428

Overholt WA, Ochieng JO, Lammers P, Ogedah K (1994) Rearing and field release methods for Cotesia flavipes Cameron (Hymenoptera: Braconidae), a parasitoid of tropical gramineous stem borers. Insect Sci Appl 15:253-259

Pettersson J (1970) An aphid sex attractant 1. Biological studies. Entomol Scand 1:63-73

Powell W, Pennachio F, Poppy GM, Tremblay E (1998) Strategies involved in the location of hosts by the parasitoid Aphidius ervi Haliday (Hymenoptera: Braconidae: Aphidinae). Biol Control 11: 104-112

Slabbert O, Van den Berg J (2009) The effect of the adjuvant, Break-Thru S240, on whorl penetration and efficacy of foliar insecticide applications against Chilo partellus. S Afr J Plant Soil 26:254-258

Takabayashi J, Dicke M, Posthumus M (1991) Variation in composition of predator-attracting allelochemicals emitted by herbivore-infested plants: relative influence of plant and herbivore. Chemoecology 2:1-6

Tamiru A, Bruce TJA, Woodcock CM, Caulifield CJ, Midega CAO, Ogol CKPO, Mayon P, Birkett MA, Pickett JA, Khan ZR (2011) Maize landraces recruit egg and larval parasitoids in response to egg deposition by a herbivore. Ecol Lett 14:1075-1083

Tamiru A, Bruce TJA, Midega CAO, Woodcock CM, Birkett MA, Pickett JA, Khan ZR (2012) Oviposition induced volatile emissions from African smallholder farmers' maize varieties. J Chem Ecol 38: $232-234$

Turlings TCJ, Tumlinson JH, Lewis WJ (1990) Exploitation of herbivoreinduced plant odors by host-seeking parasitic wasps. Science 250 : $1251-1253$

Turlings TCJ, Bernasconi M, Bertossa R, Bigler F, Caloz G, Dorn S (1998) The induction of volatile emissions in maize by three herbivore species with different feeding habits-possible consequences of their natural enemies. Biol Control 11:122-129

Wadhams LJ (1990) The use of coupled gas chromatography: electrophysiological techniques in the identification of insect pheromones. In: McCaffery AR, Wilson ID (eds) Chromatography and isolation of insect hormones and pheromones. Plenum, New York, pp 289 298

Zakir A, Sadek MM, Bengtsson M, Hansson BS, Witzgall P, Anderson P (2013) Herbivore-induced plant volatiles provide associational resistance against an ovipositing herbivore. J Ecol 101:410-417 